same temperature. Two determinations were made on each sample and the average value was reported. Analyses were made on a General Electric XRD- 5 X-ray diffractometer using iron-filtered cobalt $K \alpha$ radiation and a scanning speed of $1 / 5^{\circ} / \mathrm{min}$. The angular location of the peaks was determined as the midpoint of the half-height line. When peaks from different reflections were overlapping they were resolved graphically. Single peaks were determined to $0.01^{\circ}$, but precision was generally not as good for overlapping peaks. The lattice parameters were calculated on an IBM 7094 computer using the Argonne National Laboratories B 106 program.

Results of the calculations are given in Table I. Although the changes are at the limit of precision of the data, there is a slight decrease in the volume of the unit cell when the zirconia is oxygen-deficient. It is believed that a decrease indicates that there is oxygen missing rather than additional $\mathrm{Zr}$ in the lattice. In addition, the zirconia phase in the $\mathrm{Zr}+$ $50 \%$ o composition has the smallest unit cell, and this is consistent since it is the most deficient in oxygen.

\section{Summary}

Studies were made in the system $\mathrm{Zr}-\mathrm{O}$ in the composition range 50 to $66.7 \% \mathrm{O}$ using metallographic and high-temperature X-ray analysis. Results of this work established the solubility of zirconium in zirconia from $1200^{\circ}$ to $2000^{\circ} \mathrm{C}$, and the cubic-tetragonal inversion temperature at approximately $1490^{\circ} \mathrm{C}$ for $\mathrm{ZrO}_{2}$ solid solution in the binary system. Also, the orientation of the striations formed during the cubictetragonal inversion was studied by $\mathrm{X}$-ray analysis, and data taken at room temperature indicate that the striations are oriented with the 111 and $11 \overline{1}$ planes of monoclinic zirconia.

\section{Acknowledgments}

The authors express sincere appreciation to E. J. Hassell, N. M. Tallan, H. A. Lipsitt, and T. J. Rockett for many helpful
Table I. Lattice Parameters and Unit Cell Volumes of Zirconia in Fired and Unfired Compositions

\begin{tabular}{|c|c|c|c|c|c|}
\hline $\begin{array}{c}\text { Composition and } \\
\text { heat treatment }\end{array}$ & (A) & $\stackrel{b}{(A)}$ & $\left(\begin{array}{c}c \\
(\mathrm{~A})\end{array}\right.$ & $\stackrel{8}{\left({ }^{\circ}\right)}$ & $\underset{\left(\mathrm{A}^{2}\right)}{V}$ \\
\hline \multirow{4}{*}{$\begin{array}{l}\mathrm{ZrO}_{2}(\text { raw material }) \\
\mathrm{ZrO}_{2}\left(2000^{\circ} \mathrm{C}, 4 \mathrm{hr}, \mathrm{Q}\right) \\
\mathrm{ZrO}_{2}\left(1450^{\circ} \mathrm{C}, 280^{\mathrm{hr}},\right. \\
\mathrm{Q}) \\
\mathrm{Zr}+50 \% \text { O }\left(2000^{\circ} \mathrm{C},\right. \\
2 \mathrm{hr}, \mathrm{Q})\end{array}$} & & 5 & & & 140.8 \\
\hline & & & & & \\
\hline & 5.150 & 5.209 & 5.316 & 99.27 & 140.73 \\
\hline & 5.148 & 5.207 & 5.317 & 99.26 & 140.65 \\
\hline
\end{tabular}

discussions; to M. H. Mueller, Argonne National Laboratory, for permission to use the Argonne B 106 computer program for lattice parameter determinations; to E. L. Godfrey, Computer Laboratory, Wright-Patterson Air Force Base, for making the lattice parameter calculations; and to $H$. $O$. Bielstein for conducting firings and preparing samples.

\section{References}

1 R. F. Domagala and D. J. McPherson, "System ZirconiumOxygen," J. Metals, 6, Trans. AIME, 200 [2] 238-46 (1954).

2 Max Hansen and Kurt Anderko, Constitution of Binary Alloys, 2 d ed., pp. 1078-80. McGraw-Hill Book Co., New York, 1958.

${ }^{3}$ Erich Gebhardt, H. D. Seghezzi, and Wolfgang Duerrschnabel, "Research on the System Zirconium-Oxygen: I," J. Nucl. Mater ., 4 [3] 241-54 (1961); "II," pp. 255-68; "III," pp. $269-71$

${ }^{4}$ D. K. Smith and C. F. Cline, "Verification of Existence of Cubic Zirconia at High Temperature," J. Am. Ceram. Soc., 45 [5] $249-50$ (1962).

5 (a) R. W. Vest, N. M. Tallan, and W. C. Tripp, "Electrical Properties and Defect Structure of Zirconia: I," ibid., 47 [12] 635-40 (1964).

(b) R. W. Vest and N. M. Tallan, "Electrical Properties and Defect Structure of Zirconia: II," ibid., 48 [9] 472-75(1965).

\title{
Optical and Microstructural Variations with Electric Field in Barium-Strontium Titanate Ceramics
}

\author{
VIOLA C. SANVORDENKER \\ Microelectronics Laboratory, The University of Michigan, Ann Arbor, Michigan 48104
}

\begin{abstract}
Quasi-thin sections of large-grained ferroelectric barium-strontium titanate ceramic compositions were observed under crossed polars. Dielectric constant and light transmission were measured as a function of temperature and applied dc voltages. Microscopic observations, such as changes in structure and in optical anisotropy with temperature and simultaneously applied electric fields, are described. Optical data indicate that static electric fields readily induce anisotropy in the isotropic grains at the ferroelectric to paraelectric transition of the ceramic. Below the transition temperature, the effect of
\end{abstract}

Received May 23, 1966; revised copy received December 5, 1966.

The writer is a research associate, Microelectronics Laboratory, Electrical Engineering Department, The University of Michigan. the field on the optical and dielectric properties diminishes. Contrary to single-crystal barium titanate, a field-produced reorientation of domains in the ceramic at temperatures below the transition proceeds with difficulty, and is sometimes achieved only after long exposures to highbiasing fields.

\section{Introduction}

Prical observations on ferroelectric ceramics, such as polycrystalline barium titanate and barium-calcium titanate, have been described by a number of authors. ${ }^{1-8}$ In most cases the metallographic techniques of polishing and etching were used to examine the microstructure of the ceramic. The investigators revealed that the ceramic ferroelectric surfaces, despite their complexity, have domain configurations similar to those found in single-crystal barium 
Table I. Composition and Dielectric Data for Seven Barium-Strontium Titanate Ceramics

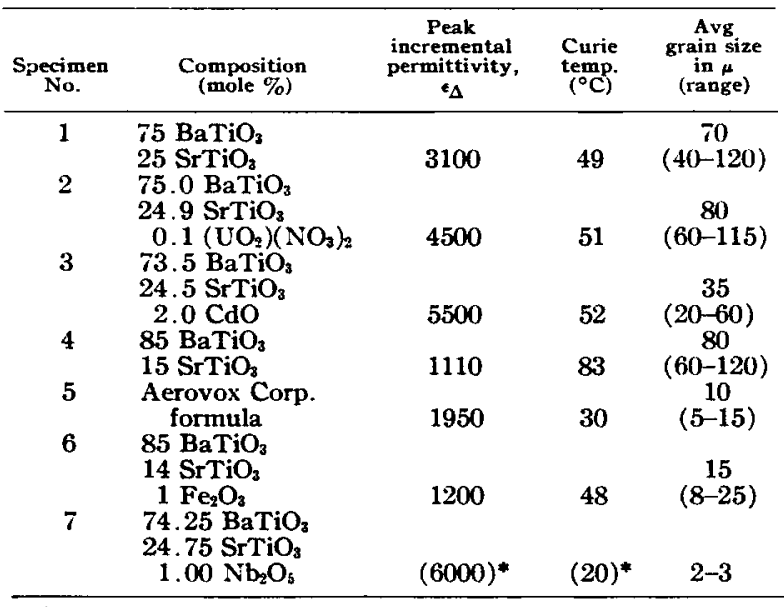

* Value obtained from larger ceramic sample of the same composition. Specimen 7 , used for transmitted light intensity measurements, had too low a capacitance for accurate determination.

titanate. The present paper describes observations made on quasi-thin sections of ferroelectric barium-strontium titanate ceramic, viewed in transmitted, polarized light. Optical and structural variations in the ceramic are described resulting from changes in temperature and applied electric field in the ferroelectric to paraelectric transition region of the ceramic compositions. It is shown that ceramic barium-strontium titanate compositions exhibit certain similarities with singlecrystal barium titanate in their optical and dielectric behavior. Field-produced domain motion, however, appears to proceed with considerably more difficulty in the ceramic ferroelectric material than in single crystals.

The present paper is condensed from an earlier, extensive study on the effects of temperature and biasing fields on the optical properties of perovskite-type ceramics. ${ }^{9}$

\section{Experimental Procedure}

Ceramic slabs, 2.5 by 1.2 by $0.25 \mathrm{~cm}$ were prepared according to the standard ceramic techniques of pressing, sintering to maturity, and furnace cooling. The starting materials were high purity commercial barium titanate and strontium titanate powders* and reagent grade oxide additives. The compositions and electrical data of the specimens are listed in Table I. All ceramics were fired at $1380^{\circ} \mathrm{C}$ for $2 \mathrm{hr}$. Grain size variation was extensive (Table I). The densities varied between 5.5 and 5.8. The addition of small percentages of metal oxides generally produced ceramics of higher densities. The fired ceramic pieces were shaped into narrow, wedgelike specimens about $0.8 \mathrm{~cm}$ long and $0.1 \mathrm{~cm}$ wide, having a thickness that tapered from 0.1 to $0.004 \mathrm{~cm}$ from end to end. The wedge-shaped geometry made possible simultaneous optical observation and capacitance measurements of the ceramic. Previous measurements of capacitance of thin sections were subject to inaccuracies because of the low values of the specimen. A section 1 by 0.8 by $0.004 \mathrm{~cm}$ typically measured only 1 to $2 \mathrm{pf}$, hence the bulkier wedge shape of approximately $30 \mathrm{pf}$ was adopted.

The specimens were prepared by embedding the ferroelectric material in thermosetting resin and grinding on a metallographic wheel, using a series of graded silicon carbide papers. The final polish was done on a fine diamond wheel

\footnotetext{
* Supplied by the Titanium Alloy Manufacturing Division National Lead Company, Niagara Falls, New York.
}

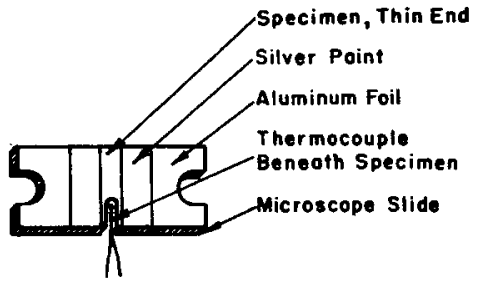

Fig. 1. Ceramic wedge mounted for observation by transmitted light. (particles of $6 \mu$ ) using methyl alcohol as a lubricant. The ceramic was then removed from the resin, cut into a narrow rectangle, and glued to a microscope slide with the polished side down. Since the thin end of the specimen was to be observed microscopically, a polymerizing cement with a refractive index near that of glass was used as an adhesive. ${ }^{10}$ The mounted specimen was then reembedded in the thermosetting resin, thereby exposing the unpolished side, and ground into the shape of a wedge. After a final polish on the diamond wheel, the specimen was lifted out of the resin with its glass backing and prepared for electrical and optical measurements. A combination of low resistance silver paint along the edges of the wedge and aluminum foil (Fig. 1) provided electrical contact for the capacitance measurements. These were taken at $1600 \mathrm{kc}$ on a Boonton Radio Corporation Q-Meter. A $1300 \mathrm{v}$ dc supply furnished the required voltages to the specimen. The wedge was mounted over a small heating stage made of Nichrome resistance wire and asbestos (Fig. 2) and clipped to the stage of a Leitz Panphot microscope. A thermocouple was placed in a slot directly beneath the wedge. As the specimen was alternately heated and cooled through a range of approximately $100^{\circ} \mathrm{C}$, the thin end of the ceramic was observed through transmitted light and crossed polars. Simultaneously, capacitance measurements were taken while biasing fields ranging between 0 and $20,000 \mathrm{v} / \mathrm{cm}$ were supplied to the specimen. The dc field to the ceramic was applied parallel to the plane of the microscope stage. Thus any alignment of the polar vector in a ferroelectric grain by an electric field would become observable as a change in the anisotropy of that grain.

In addition to the direct observation of optical and structural details in the ceramic, the light intensity transmitted by the thin end through crossed polars was measured as a function of temperature and field. A CdS photocell, mounted over the microscope eyepiece, registered the total light transmitted by the grains lying in the field of the microscope. The photocurrent readings taken on the ceramic were compared with readings taken on a calibrated Kodak density film strip.

\section{Data}

Seven compositions of $(\mathrm{Ba}, \mathrm{Sr}) \mathrm{TiO}_{3}$, some with metal oxide additives, were prepared and examined as described in Section II. Ceramic and dielectric data are listed in Table I 


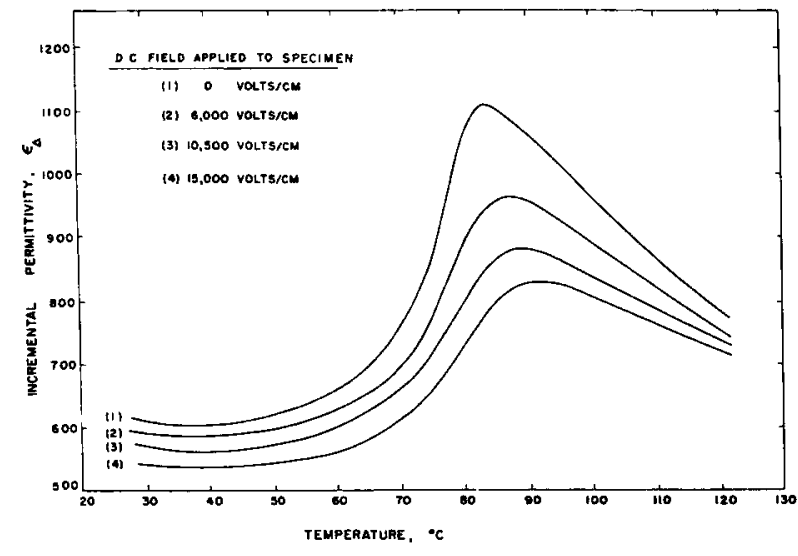

Fig. 3. Incremental permittivity vs. temperature of $\left(\mathrm{Ba}_{0.85}\right.$ $\left.\mathrm{Sr}_{0.15}\right) \mathrm{TiO}_{3}$ with voltage as a parameter.

The specimens are composed of grains varying from $\overline{5}$ to $120 \mu$ in diameter. The dielectric data of all specimens show the same general behavior with temperature and field. The optical light transmittancy readings for the larger grained* compositions, however, are approximately four times that of the fine-grained ceramics in their anisotropic region. This seems reasonable when one considers the larger amount of scattering and absorption occurring when light passes through a $30 \mu$ section composed of several layers of 1 to $5 \mu$ grains rather than one layer of 50 to $100 \mu$ grains.

The dielectric and optical data for a representative, large-grained specimen of $\mathrm{Ba}_{0.85} \mathrm{Sr}_{0.15} \mathrm{TiO}_{3}$, approximately $80 \mu$ in diameter on the average, are shown in Figs. 3 and 4 . Figure 3 illustrates the behavior of the incremental, smallsignal $\dagger$ permittivity, $\epsilon_{\Delta}$, as a function of temperature and biasing fields. Figure 4 shows the changes in the percentage of light transmitted by the thin end of the specimen as a function of temperature and electric field. The dielectric and optical data were taken simultaneously. The discrepancy in the values of the applied de fields in Figs. 3 and 4 arises from different specimen widths used in the computation. For the incremental permittivity curves the biasing field was obtained by considering the average width of the wedge across which the voltage was applied. For the percent light transmission curves the field was calculated for the actual width at the thin end of the wedge. It may be argued that the general behavior at the thin section end of the ceramic is not entirely representative of the entire specimen. This is undoubtedly true since the thin end of a large-grained $(\mathrm{Ba}, \mathrm{Sr})$ $\mathrm{TiO}_{3}$ ceramic is essentially composed of a single layer of grains and it is consequently not as highly strained as the three-dimensional array of grains in the bulk ceramic. Nevertheless, a comparison of the electrical and optical data of Figs. 3 and 4 indicates that the ferroelectric to paraelectric transition for a given biasing field occurs at approximately the same temperature for both capacitance and light transmittancy measurements. A comparison of the dielectric and optical properties therefore seems permissible on a qualitative basis.

Figure 3, curve 1 (ceramic with zero bias), shows the characteristic "peak" in the incremental permittivity which is associated with a phase change in ferroelectric ceramics, in

\footnotetext{
* "Large grained" here pertains to grains having a diameter * "Large grained" here pertains to grains having a diameter
larger than the thickness of the observed ceramic section, i.e. 30 to $40 \mu$, and "fine grained" pertains to those having diameters smaller than the thickness of the section.

$t$ The incremental permittivity, $\epsilon \Delta$, is the permittivity $d P / d E$ obtained by measuring the dielectric with a small, high-frequency signal superimposed on a large dc biasing field.
}

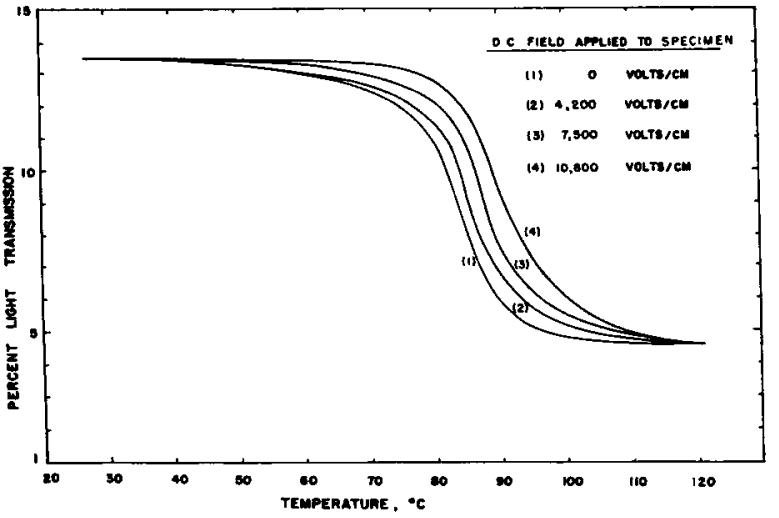

Fig. 4. Percent light transmission vs. temperature of $\left(\mathrm{Ba}_{0.85}-\right.$ $\left.\mathrm{Sr}_{0.15}\right) \mathrm{TiO}_{3}$ with voltage as a parameter.

this case the tetragonal to cubic transition. It is seen in Fig. 3 , curves 2,3 , and 4 that the peak incremental permittivity shifts to lower values and to higher temperatures as the biasing field is increased. The percent light transmission vs. temperature curves for the same ceramic likewise show a shift to higher temperatures with increasing applied dc voltages. The transmitted light intensity of the specimen experiences a sharp drop in the transition region of the ceramic, since a large number of the individual ceramic grains, which have passed from an anisotropic to isotropic state, no longer rotate the light out of the plane of the crossed polars. When the ceramic is exposed to strong dc fields, however, ferroelectricity is induced ${ }^{11}$ in some isotropic grains and the anisotropic state persists to higher temperatures. As a result of this behavior, it is possible to produce relatively large changes in optical transmittance by biasing the ceramic at the transition region. For instance, the light transmission for $\left(\mathrm{Ba}_{0.85}-\right.$ $\left.\mathrm{Sr}_{0.15}\right) \mathrm{TiO}_{3}$ changes from 7 to $11 \%$ at $87^{\circ} \mathrm{C}$ for a field of 11,000 $\mathrm{v} / \mathrm{cm}$. By comparison the transmission with field increases only from 13 to $13.5 \%$ at $60^{\circ} \mathrm{C}$ where the ceramic is ferroelectric (see Figs. 3 and 4). Similar changes in the "transparency" with biasing fields have been measured by Kawabe'12 on single-crystal barium titanate at the Curie point. The capacitive nonlinearity with field of these ceramics has been successfully used in the past in modulating devices and amplifiers. It seems that the corresponding optical nonlinear properties of transparent "ceramic" films could lead to the development of some useful electrooptic devices.

In the following, microscopic observations on the ceramic are described, emphasizing structural changes in individual grains resulting from variations in electric field and temperature.

\section{Observations}

A number of changes noted in the microstructure of the ceramic during the capacitance and light transmission measurements are classified roughly as short-term and long-term effects. Short-term effects are the optical and structural changes in the ceramic that follow immediately on exposure to an external dc field and vanish when the field is removed. Long-term effects require a prolonged exposure $(20 \mathrm{~min})$ to an external dc field. The resulting changes persist in the ceramic microstructure after the field has been removed. These latter effects can also be considered "poling" or "polarization" effects.

\section{(1) Short-Term Effects}

When a strong biasing field is applied to a thin wedge, a number of optical changes observed in the ceramic depend on the temperature at which the field is applied. At about $50^{\circ} \mathrm{C}$ 


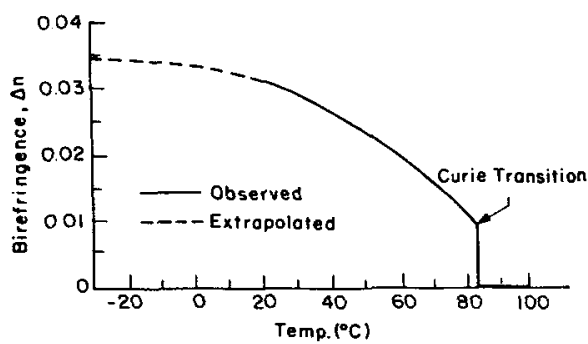

Fig. 5. Variation of birefringence with temperature in $\left(\mathbf{B a}_{0.85} \mathbf{S r}_{0.15}\right) \mathrm{TiO}_{\mathbf{8}}$ ceramic

above the temperature of the peak incremental permittivity, a ceramic consists of essentially isotropic grains, and the "thin section" remains dark under crossed polars as expected. Strong biasing fields applied to the specimen at this temperature do not produce optical anisotropy. The large changes observed in transmitted intensity occur in the ferroelectric to paraelectric transition region where a grain may go from complete extinction to a bright first order white when the field is applied. Again, substantially below the peak permittivity temperature (i.e. about $50^{\circ} \mathrm{C}$ ), little optical change as a result of applied field is observed in the now highly birefringent ferroelectric grains. Most optical as well as structural changes in the ferroelectric grains occur within $30^{\circ} \mathrm{C}$ below the peak incremental permittivity temperature. Considerable differences exist in the degree of observable optical change with field among several barium-strontium titanate compositions. The description in the following is limited to the larger-grained ceramics since no birefringence colors or fine structural detail could be observed in the fine-grained ceramic with the Leitz Panphot microscope. Ceramics having an average grain size of $10 \mu$ or less, however, showed patches of residual anisotropy, even when heated far beyond the transition temperature. Such anisotropy must be attributed to the existence of strained areas in the finer-grained material.

(A) Birefringence as a Function of Temperature and Electric Field: As illustrated in Fig. 5, the birefringence of a $\left(\mathrm{Ba}_{0.85} \mathrm{Sr}_{0.15}\right) \mathrm{TiO}_{3}$ specimen decreases with increasing temperature, similar to that of a single crystal of barium titanate. A grain $30 \mu$ thick changed from a second order green at room temperature to a first order white at the peak incremental permittivity temperature, $83^{\circ} \mathrm{C}$. Hence the birefringence decreases from 0.03 to 0.01 over a $60^{\circ} \mathrm{C}$ range. A field of $10,000 \mathrm{v}$ applied across the specimen at room temperature produces a change from a second order green to a second order yellow. This represents an increase in anisotropy of approximately 0.005 for the specimen at that temperature.

(B) Structural Changes Due to Temperature and Electric Field: The structural changes in the ceramic grains which result from temperature and electric field variations are visible in those grains which show parallel twinning striations $\left(90^{\circ}\right.$ domains). As a rule, the twinning striations in the grains become diffuse as the specimen is heated to the peak incremental permittivity temperature, until the entire grain appears a uniform white or gray color and finally becomes isotropic; however, when strong fields are applied near the transition temperature, some twin lines reappear in their original direction and number. This may be interpreted as an elongation of the polar axis by the field so as to increase the $c / a$ axial ratio and the tetragonal nature of the grain.

\section{(2) Long-Term Effects}

Considerable optical change in the grains of a ferroelectric barium-strontium titanate specimen can be observed when a strong biasing field is applied to the specimen for 20 to $30 \mathrm{~min}$. The field of view becomes brighter immediately after the field is applied. Then, with time, photocell readings of the

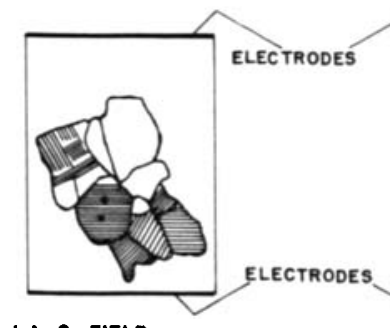

(a) O-FIELD

(b) 17,000 VOLTS/CM, FOR 20 MINUTES

$$
\begin{aligned}
& \text { INTERFERENCE COLORS } \\
& \text { YELLOW, WHITE, } \\
& \text { GRAY }
\end{aligned}
$$

Fig. 6. Poling in a $\left(\mathrm{Ba}_{0.75} \mathrm{Sr}_{0.25}\right) \mathrm{TiO}_{3}$ ceramic, at room temperature.

transmitted light intensity show a gradual increase and subsequent saturation of the current. This is accompanied by an increase in interference colors of isolated grains. In addition, grains which have twinning lamellas may show lasting changes in the direction of these lamellas after prolonged exposure to the electric field. A short (1 to $2 \mathrm{sec}$ ) exposure to the field causes either temporary fading or intensification of twinning striations depending on the relative orientation of the applied field to the polarization direction in the grains. Long exposures (up to $30 \mathrm{~min}$ ) to a strong field, however, may produce local changes in both interference colors and twinning striations which remain after the field is removed (Fig. 6).

\section{Summary}

The dielectric and optical behavior of $(\mathrm{Ba}, \mathrm{Sr}) \mathrm{TiO}_{3}$ ceramics, of which several examples have been given, is summarized below:

(1) $(\mathrm{Ba}, \mathrm{Sr}) \mathrm{TiO}_{3}$ ceramic, just as single-crystal $\mathrm{BaTiO}_{3}$, exhibits a peak in the dielectric properties as the ceramic undergoes a phase change from tetragonal, ferroelectric, to cubic, paraelectric. The transition in the ceramic, however, takes place over a wider temperature range due to intergranular and intragranular strains which affect the Curie temperature of the individual grains. Moreover, the addition of strontium titanate to lower the Curie temperature of pure barium titanate results in some compositional inhomogeneity in the grains of the resulting solid solution. This inhomogeneity causes a further spread of the transition temperature. Piezoelectric ceramic manufacturers make use of the broadened dielectric peak to produce less-temperaturesensitive materials at the expense of a lower dielectric constant.

(2) Within the temperature interval of the transition it is possible to effect a large decrease in incremental permittivity and to noticeably increase the transmitted light intensity by applying a high dc voltage to the specimen. Both dielectric and optical phenomena are linked to a field-induced ferroelectric-anisotropic state in the ceramic. ${ }^{11}$ Such an effect exists in single crystals of $\mathrm{BaTiO}_{3}$ near the Curie temperature. ${ }^{13}$ As a consequence of the induced ferroelectric effect, the peak incremental permittivity temperature of a biased specimen is higher than that of the specimen at zero bias.

(3) The optical anisotropy of $(\mathrm{Ba}, \mathrm{Sr}) \mathrm{TiO}_{3}$ ceramics in their tetragonal, ferroelectric state is temperature dependent. Large-grained (50 to $100 \mu$ ) ceramic specimens increase in birefringence with decreasing temperature. For an equivalent temperature interval, the change in birefringence in the ceramic is less than in single-crystal barium titanate.

(4) The optical anisotropy of ferroelectric barium-strontium titanate ceramics is also field dependent. The application of a dc voltage instantaneously changes the birefringence 
colors in numerous grains to higher orders. This increase in anisotropy appears to result from field-produced strains rather than from domain reorientation, for the twinning striations do not generally change direction immediately after a field is applied. Near the transition temperature, the field-produced optical anisotropy is more pronounced.

(5) Although short-term field effects do not produce identifiable signs of domain switching, the continuous application, below the transition temperature, of a strong de field for up to $30 \mathrm{~min}$ may give rise to poling in some ceramic specimens. The new birefringence colors and twinning patterns formed persist after the field is removed.

(6) For purposes of optical investigation, several compositions had to be studied with grain sizes larger than desired for an optimum industrial ceramic. In general, the largegrained (50) to 12()$\left._{\mu}\right)$ compositions were more susceptible to poling than the fine-grained ceramics ( 2 to $15 \mu$ ). Strained areas, on the other hand, increased as the size of the ceramic grain decreased. When the grain size is below $100 \mathrm{~A}$, the ferroelectric to paraelectric transition becomes increasingly diffuse and the ceramic loses its piezoelectric characteristics. Thus correct grain size becomes an important factor in the electrical stability of barium-strontium titanate and similar piezoelectric ceramics.

\section{Acknowledgments}

The author thanks Howard Diamond, under whose direction the present study was conducted, for his sustained interest and encouragement, and R. M. Denning for numerous consultations on optical techniques and development of a modified thin section configuration.
References

1 O. Ruediger, "Microscopic Evidence of Domain Structure in Polycrystalline Barium Titanate," Naturvissenschaften. 42 [24] 644 (1955).

${ }^{2}$ W. R. Cook, Jr., "Domain Twinning in Barium Titanate Ceramics," J. Am. Ceram. Soc., 39 [1] 17-19 (1956).

${ }^{3}$ Frank Kulcsar, "A Microstructure Study of Barium Titanate Ceramics," ibid., pp. 13-17.

${ }^{4} \mathrm{D}$. Kontoleon and J. Tomlinson, "Micro-Examination of Barium Titanate," Metal Progr., 69 [5] 98-102 (1956).

${ }^{5}$ R. C. DeVries and J. E. Burke, "Microstructure of Barium Titanate Ceramics," J. A m. Ceram. Soc., 40 [6] 200-206 (1957). ${ }^{B}$ G. L. Pearson and W. L. Feldmann, "Powder-Pattern Techniques for Delineating Ferroelectric Domain Structures," J. Phys. Chem. Solids, 9 [1] 28-30 (1959).

7 V. J. Tennery and F. R. Anderson, "Examination of the Surface and Domain Structure in Ceramic Barium Titanate," J. Appl. Phys., 29 [5] 755-58 (1958).

8 J. de Boer, "Microscopic Investigation of Domain Structure in Polycrystalline Barium Titanate," Chem. Weekblad, 54 [11] $137-41$ (1958)

9 V. C. Sanvordenker, "Optical Observation by Transmitted Light of the Tetragonal to Cubic Phase Transition in BariumStrontium Titanate," The University of Michigan, Industry Program, IP 652, January 1964. Univ. Microfilms (Ann Arbor. Mich.), Order No. 64-8205, 190 pp.; Dissertation Abstr., 25 [4] 2550 (1964).

${ }_{10} \mathrm{~F}$. H. Andres and J. H. Richardson, "New Cement for Preparation of Ceramic Thin Sections," J. Am. Ceram. Soc., 43 [5] $282(1960)$.

II Howard Diamond, "Variation of Permittivity with Electric Field in Perovskite-Like Ferroelectrics," J. Appl. Phys., 32 [5] 909-15 (1961).

12 Kazuo Kawabe, "Optical Studies on the Effect of Electric Fields on the Transitions of Barium Titanate," J. Phys. Soc. Japan, 14 [12] 1755-65 (1959).

${ }^{13}$ W. J. Merz, "Double Hysteresis Loop of Barium Titanate at the Curie Point," Phys. Rev., 91 [3] 513-17 (1953).

\title{
Lower-Temperature Modifications of $\mathrm{Nb}_{2} \mathrm{C}$ and $\mathrm{V}_{2} \mathrm{C}$
}

\author{
ERWIN RUDY and CHARLES E. BRUKL
}

Materials Research Laboratory, Aerojet-General Corporation, Sacramento, California 95809

\begin{abstract}
The orthorhombic, low-temperature $\alpha$-modification of $\mathrm{Nb}_{2} \mathrm{C}$ has a structure similar to that of $\zeta-\mathrm{Fe}_{2} \mathrm{~N}\left(a=12.36 \mathrm{~A}, b=10.89_{\mathrm{b}} \mathrm{A}, c=4.96_{8} \mathrm{~A}\right)$ and transforms at $\sim 1200^{\circ} \mathrm{C}$ into the hexagonal $\mathrm{C}_{-\mathrm{Fe}} \mathrm{N}$ type. $A$ second transition at approximately $2500^{\circ} \mathrm{C}$ is associated with the destruction of long range order in the carbon sublattice. Alpha-divanadium carbide (orthorhombic, $a=$ $11.49 \mathrm{~A}, b=10.06 \mathrm{~A}, c=4.55 \mathrm{~A}$ ) is isostructural with $\alpha-\mathrm{Nb}_{2} \mathrm{C}$ and transforms at $\sim 800^{\circ} \mathrm{C}$ into the hexagonal high-temperature modification. The structures of $\alpha-V_{2} \mathrm{C}$ and $\alpha-\mathrm{Nb}_{2} \mathrm{C}$ are distorted modifications related to the $\epsilon-\mathrm{Fe}_{2} \mathrm{~N}$ type.
\end{abstract}

\section{Introduction}

$I^{\mathrm{x}}$ CONTRAST to the previously assumed $L^{\prime} 3$ structure for the $\mathrm{Me}_{2} \mathrm{C}$ phases of the refractory transition metals, ${ }^{1}$ recent neutron and electron diffraction studies revealed an essentially ordered distribution of the carbon atoms among the interstitial lattice sites. According to Parthé and Sadagopan, ${ }^{2} \mathrm{MO}_{2} \mathrm{C}$ is only pseudohexagonal; the true structure is orthorhombic (space group $D_{2 h}^{14}-P b c n, \zeta-\mathrm{Fe}_{2} \mathrm{~N}$ type). The dimensions of the supercell are related to the hexagonal axes by approximately $a_{0}=c_{H}, b_{0}=2 a_{H}$, and $c_{0}=a_{H} \sqrt{3}$. Di- tungsten carbide ${ }^{3}$ and $\mathrm{Ta}_{2} \mathrm{C}^{4}$ are hexagonal and correspond to the $C 6$ type $\left(\mathrm{CdI}_{2}\right.$ antitype). The sublattice order of $\mathrm{Nb}_{2} \mathrm{C}^{5}$ $\left(\epsilon-\mathrm{Fe}_{2} \mathrm{~N}\right.$ type) is similar to that of $\mathrm{Mo}_{2} \mathrm{C}$, whereas the structure of $\mathrm{V}_{2} \mathrm{C}$ was claimed to be of the disordered $L^{\prime} 3$ type. ${ }^{4}$

In investigations on binary and ternary transition metalcarbon systems, ${ }^{6}$ observed high-temperature phase transformations of the subcarbide phases were associated with orderdisorder reactions occurring in the semimetal sublattices of these compounds.7.8 The type of transformation involved varies with the specific carbide. In the Group VIB metal subcarbides, disordering proceeds as a homogeneous reaction at hypostoichiometric compositions, whereas hyperstoichiometric alloys undergo a discontinuous phase change ${ }^{9.10}$; i.e. within a certain concentration range two phases coexist with the same structural framework of the metal host lattice, but differing in the lattice dimensions and in the degree of order in the carbon sublattice. In the Group VB metal-carbon

Received September 14, 1966; revised copy received December 5,1967 .

This work was supported by the Air Force Materials Laboratory, Research and Technology Division, Air Force Systems Command, Wright-Patterson Air Force Base, under Contract No. AF 33(615)-1249.

The writers are, respectively, section head and senior chemist. Materials Research Laboratory, Aerojet-General Corporation. 